\title{
Multiwavelength observations of the field HS 47.5/22 in Ursa Major ${ }^{\star, \star \star}$
}

\section{The X-ray catalogue of a medium deep ROSAT survey}

\author{
K. Molthagen ${ }^{1}$, H.J. Wendker ${ }^{1}$, and U.G. Briel ${ }^{2}$ \\ 1 Hamburger Sternwarte, Gojenbergsweg 112, D-21029 Hamburg, Germany \\ 2 Max-Planck-Institut für extraterrestrische Physik, D-85740 Garching, Germany
}

Received March 10; accepted April 17, 1997

\begin{abstract}
We present the X-ray source catalogue obtained from a ROSAT survey in the HQS field HS 47.5/22. The survey consists of 48 overlapping PSPC pointings which were first analysed individually, and then merged to increase the sensitivity. Both modes bring forth sources missed in the other, showing that both are necessary to detect all X-ray sources in the field. The final catalogue contains $574 \mathrm{X}$-ray sources. The identifications are mainly based on the HQS objective prism plate which allows object classification down to a magnitude $m_{\mathrm{b}} \lesssim 18 \mathrm{~m} .5$, and a rough distinction between red and blue objects more than a magnitude deeper. Follow-up observations were performed for a number of objects, mainly faint quasar candidates.
\end{abstract}

Key words: methods: data analysis - catalogues surveys $-\mathrm{X}$-rays: general $-\mathrm{X}$-rays: galaxies

\section{Introduction}

Although this work is primarily interested in X-ray emission, especially of AGN, the project started with a photographic plate from the Hamburg Quasar Survey (HQS). The HQS is an objective prism plate based survey of the

Send offprint requests to: K. Molthagen, kmolthagen@hs.unihamburg.de

* Partly based on observations from the German-Spanish Astronomical Center, Calar Alto, operated by the Max-PlanckInstitut für Astronomie, Heidelberg, jointly with the Spanish National Commission for Astronomy.

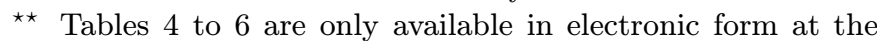
CDS via anonymous ftp to cdsarc.u-strasbg.fr (130.79.128.5) or via http://cdsweb.u-strasbg.fr/Abstract.html. Figure 7 is published electronically and is made available at http://www.edphys.fr/Abstract.html northern sky. Additional direct plates were taken for exact position determination. A detailed description can be found in Hagen et al. (1995), and a first list of quasars will be given in Engels et al. (1997).

It is obvious that the magnitude limited samples obtained as test samples for the automated quasar search are of astronomical interest as well. They fill in the magnitude gap between deep surveys which cover necessarily only small areas and extended surveys which are cut off at high magnitudes.

The field HS $47.5 / 22$, centred around $9^{\mathrm{h}} 50^{\mathrm{m}} / 47^{\circ} 30^{\prime}$ (B1950.0), at a galactic latitude of $\sim 50^{\circ}$ was selected for follow-up observations in several wavelength ranges. The selection was based solely on the extraordinary plate quality. Telescope condition, weather, and plate material, were all at their best when the plates were taken, which allows the classification of objective prism spectra, and the recognition of optical counterparts on the direct plate, to much fainter magnitudes than the average HQS field. This "technical" selection holds the advantage of a total random choice regarding astronomical properties.

Another major advantage of the field is its location in an area with very low column density of galactic neutral hydrogen $N_{\mathrm{H}}$. Low resolution surveys like the Stark Survey (Stark et al. 1992) give an average value of $\left\langle N_{\mathrm{H}}\right\rangle=10^{20} \mathrm{~cm}^{-2}$, corresponding to a visual extinction of only 0.05 . This is not much higher than the values in the so called "Lockman Hole" which contains the lowest $N_{\mathrm{H}}$ of the whole northern sky: $N_{\mathrm{H}}=4.510^{19} \mathrm{~cm}^{-2}$ (Lockman et al. 1986). Absorption of X-rays in the interstellar medium will therefore be a comparatively minor problem.

Previous X-ray surveys have shown that the majority of the faint sources outside the galactic plane are AGN, or at least extragalactic, the fraction growing with galactic latitude (Boyle et al. 1994). Therefore the high galactic 
latitude of HS $47.5 / 22$, together with the low $N_{\mathrm{H}}$, predestinate the field for a search of extragalactic $\mathrm{X}$-ray sources.

The central part $\left(\sim 11.5 \mathrm{deg}^{2}\right)$ of HS 47.5/22 was surveyed with ROSAT (Trümper 1983). The observations consist of a series of overlapping PSPC pointings, the exposure times chosen so as to give net values between those of large scale surveys (e.g. RASS, Voges 1992; EMSS, Gioia et al. 1990) and deep observations in small areas (e.g. Hasinger et al. 1993) to cover the intermediate flux range.

The $\mathrm{X}$-ray sources were identified with the aid of the HQS plates, published catalogues, and follow-up spectroscopy. The ratio of $\mathrm{X}$-ray to optical flux, $\log \left(f_{\mathrm{x}} / f_{\mathrm{v}}\right)$, played an important role since the different types of $\mathrm{X}^{-}$ ray emitters were found in the EMSS to have different, well defined ranges (Gioia et al. 1990). ROSAT observations confirmed these albeit with slight changes due to the different energy bands of the two satellites (Bade 1993).

In this paper, we present the results of the $\mathrm{X}$-ray survey. The next section presents the $\mathrm{X}$-ray data and analysis. The third section overviews some additional data in other wavelength bands. Identification of $\mathrm{X}$-ray sources is described in the fourth, and the last contains some concluding remarks.

\section{2. $\mathrm{X}$-ray data and analysis}

The X-ray survey in HS 47.5/22 consists of 48 pointings with the ROSAT PSPC (Pfeffermann et al. 1986). These pointings form a diamond shaped grid with $\Delta \alpha=180^{\mathrm{s}}$ and $\Delta \delta=30^{\prime}$. The observations were performed between April 1991 and October 1993, and an observation log is given in Table 4 . Figure 1 gives a schematic view of the field.

Most pointings were observed in more than one interval (OBI). The exposure times of single OBIs range from $\simeq 400 \mathrm{~s}$ to $\simeq 2900 \mathrm{~s} . t_{\text {exp }}$ in Table 4 is the sum of all OBIs at a given position, the distribution of $t_{\exp }$ is presented in Fig. 2.

Since the diameter of the field of view (FOV) is $2^{\circ}$, the pointings overlap noticeably, and all but the outer rim of the field is covered by several observations. Adding up all pointings gives a resulting exposure time $\geq 5000$ s for $73 \%$ of the area and more than $20000 \mathrm{~s}$ for the central $2.3 \mathrm{deg}^{2}$ (see Fig. 3).

\subsection{Source detection on individual pointings}

Source detection on the individual pointings was done with the EXSAS software package, version 93JAN (Zimmermann et al. 1993), which uses a sliding window technique. Images were accumulated in the broad $(0.1-$ $2.4 \mathrm{keV})$, hard $(0.4-2.4 \mathrm{keV})$, and soft $(0.1-0.4 \mathrm{keV})$ bands with a pixel size of $15^{\prime \prime}$.

The first step of the source detection took the background from a region directly surrounding the detection

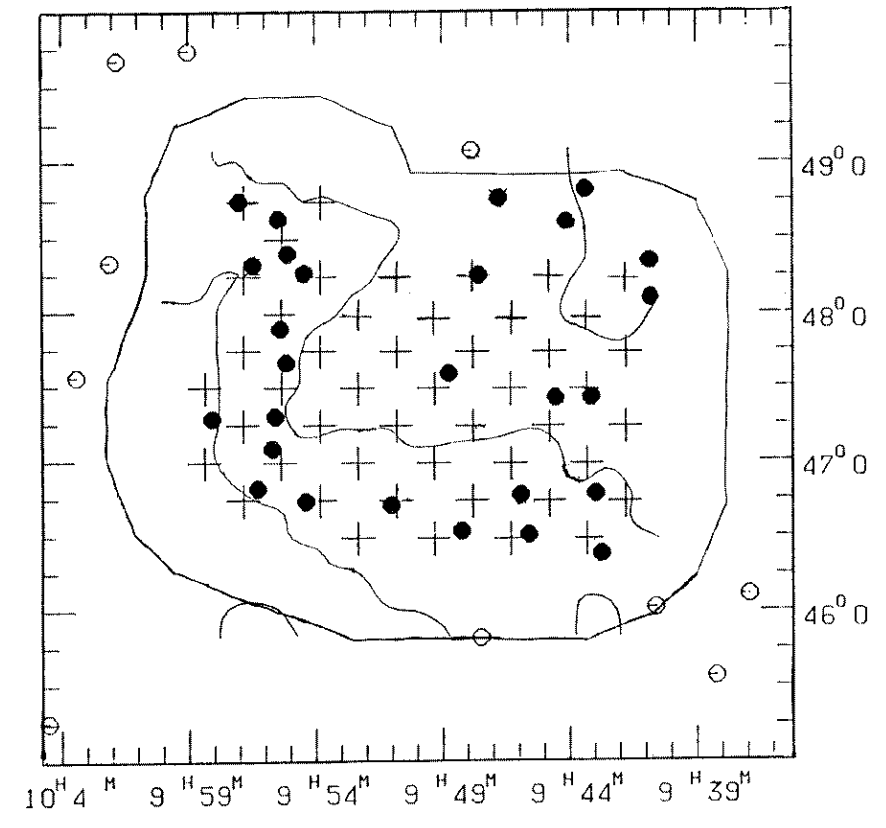

Fig. 1. Map of the field 47.5/22. Closed contour: the area surveyed with ROSAT, open contour: $N_{\mathrm{H}} \simeq 10^{20} \mathrm{~cm}^{-2}$ (Effelsberg observations), +: centres of ROSAT-pointings, $\bullet$ : optically selected quasars within the ROSAT area, o: other optically selected quasars. Coordinates are J2000.0

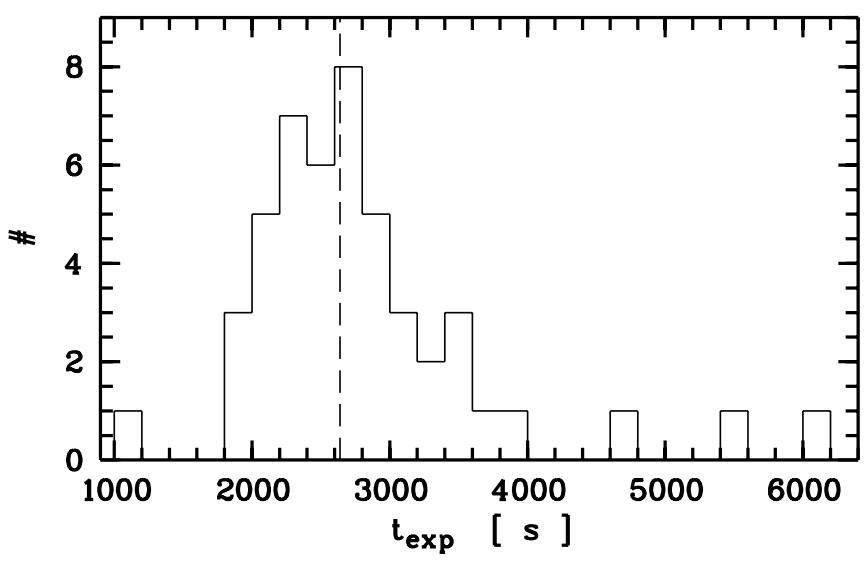

Fig. 2. Distribution of exposure times of individual pointings. The vertical line marks the median value

cell, the second took the background from the cleared and smoothed image. The third step applied a maximum likelihood analysis to the sources found previously. It computed accurate positions and their $1 \sigma$ errors, vignetting corrected source counts and count rates, and likelihood of existence, $M L_{\text {exi }}$ (Cruddace et al. 1988). $M L_{\text {exi }}=8$ was used as detection threshold; this value corresponds to a probability $P_{\mathrm{c}}=3.410^{-4}$ that the observed distribution is a pure background fluctuation, and is equivalent to a signal to noise $S / N \simeq 3$.

Each band was searched separately to allow the detection of faint sources with extreme spectra. Detections from 
different bands were considered identical if their positions differed less than $2 \sigma, \sigma$ being the larger position error. The entry with the largest $M L_{\text {exi }}$ defined the position.

After the first pointings were analysed it became clear that some sources had been missed. Most of them were located in the outer part of the FOV. An explanation would be that the detection cell in the first step had been too small, and the sources' PSF leaked into the background extraction area, causing an overestimated background and subsequent non-detection in the next steps. Although possible, this cannot be the only explanation. Tests with varying detection thresholds in the first detection step showed that the differences of the resulting background maps are almost entirely within the noise. So, background contamination is less serious than first assumed, leaving only the possibility of software failure.

Lower detection thresholds resulted in nothing but the "detection" of random fluctuations, so each pointing was inspected by eye. Undetected spots were marked and their positions used as starting point for another run of the maximum likelihood analysis. Sources above the threshold were added to the lists, about five per pointing, some of which have $M L_{\text {exi }}$ noticeably above the threshold. A repetition of the source detection with the next EXSAS version (i.e. 94JAN) on several test pointings brought forth all previously missing sources. This indicates that indeed software problems caused their non-detection.

The source lists from the various pointings were merged into a single catalogue. When the positions obtained from different pointings were compared, the $2 \sigma$ matching criterion was found to be insufficient because errors of the pointing direction itself could contribute. Therefore, two sources detected in different pointings are considered identical if their distance was less than $30^{\prime \prime}$. If the distance was between $30^{\prime \prime}$ and $45^{\prime \prime}$, and at least one source had an off-axis angle $\phi_{\text {oa }}>20^{\prime}$, they were considered to be the same as well because of the growing PSF. If at least one source was found at $\phi_{\text {oa }}>35^{\prime}$, the distance had to be larger than $55^{\prime \prime}$ before two detections were considered as two real sources. Again the entry with the highest $M L_{\text {exi }}$ determined the position. Sources found at $\phi_{\text {oa }}>40^{\prime}$ were excluded from the catalogue. The PSF is too large, and vignetting too effective, to allow the detection of any but the brightest sources, and these will presumably be blended.

Sources partially shadowed by the window support structure might show even larger $\left(>1^{\prime}\right)$ displacements and yet be multiple detections. Since these cases are very hard to parametrize, the source list was displayed on each pointing and the dubious detections were checked by eye. The resulting catalogue, later referred to as "individual catalogue", contains 489 sources.

\subsection{Merged data analysis}

\subsubsection{Merging}

Before the process of merging is started, it has to be checked whether the outer part of the FOV really contributes to enhanced sensitivity. A source detected on axis in one pointing will appear much less significant at larger $\phi_{\text {oa }}$ in other pointings. In extreme cases, merging these parts might lead to non-detection.

The average on-axis background count rate is $c r_{\mathrm{bg}}=$ $1.510^{-3} \operatorname{cts~s}^{-1} \operatorname{arcmin}^{-2}$. With that, the count rate of a source with given $S / N$ can be computed. A circular extraction area with radius $1^{\prime}$, as is appropriate for onaxis sources, and an exposure time $t_{0}=2500 \mathrm{~s}$ lead to an $S / N=5$ on-axis count rate of

$$
\begin{aligned}
c r_{\mathrm{s}, \mathrm{on}} & =\left[\frac{(S / N)^{2}}{2}+S / N \sqrt{\frac{(S / N)^{2}}{4}+\pi c r_{\mathrm{bg}} t_{0}}\right] t_{0}^{-1} \\
& =1.3510^{-2} \mathrm{cts} \mathrm{s}^{-1} .
\end{aligned}
$$

When this source is shifted to larger $\phi_{\mathrm{oa}}$, the effective exposure time is reduced due to vignetting. At $\phi_{\text {oa }}=40^{\prime}$, a reduction of a factor 0.76 is reached. Simultaneously, the PSF grows, and the extraction area has to be larger, thus containing more background. An extraction circle with 6.5 diameter is appropriate at $\phi_{\text {oa }}=40^{\prime}$. With source and background count rates as before, and $t_{40}=0.76 t_{0}$, one finds $(S / N)_{40}=$ $c r_{\mathrm{s}} t_{40} / \sqrt{t_{40}\left(c r_{\mathrm{s}}+3.25^{2} \pi c r_{\mathrm{bg}}\right)} \simeq 2.3$, clearly not detectable.

If the on-axis and off-axis detections are added together, the resulting $S / N$ is

$$
\begin{aligned}
(S / N)_{\mathrm{m}}= & {\left[c r_{\mathrm{s}}\left(t_{0}+t_{40}\right)\right] / } \\
& \sqrt{\left(c r_{\mathrm{s}}+\pi c r_{\mathrm{bg}}\right) t_{0}+\left(c r_{\mathrm{s}}+3 ! 25^{2} \pi c r_{\mathrm{bg}}\right) t_{40}} \\
\simeq & 4.3 .
\end{aligned}
$$

If an on-axis value of $S / N=3.5$ is required, the value is $(S / N)_{\mathrm{m}} \simeq 2.9$, which corresponds to the required limit of $M L_{\text {exi }}=8$, and the source is therefore barely detectable. The addition of even larger off-axis angles will lead to non-detection. In the real merged data, more than two pointings with different exposure times will usually overlap, and various $\phi_{\text {oa }}$ contribute to a certain position, so that a source is less affected than in the above estimation. However, regions with $\phi_{\text {oa }}>40^{\prime}$ will definitely not contribute to an enhanced sensitivity and are therefore removed in order to obtain a reasonable point source sensitivity. Even so, sources found close to the threshold in single pointings may be lost, depending on the actual pointings and off-axis angles which contribute to that particular position.

EXSAS provides a merging procedure for the relevant files (i.e. photon events table, eventrates table, attitude table, see Zimmermann et al. (1994) for description) which 
includes re-calculations of all sky coordinates with respect to a new common "detector centre". This procedure was applied after the outer $20^{\prime}$ of each FOV were removed.

\subsubsection{Source detection}

Source detection on the merged data was done with the 94JAN version of EXSAS (Zimmermann et al. 1994). Standard procedures were used when possible, but some adjustments were necessary.

The soft and broad bands were the same as before, whereas the hard band had changed to $0.5-2.0 \mathrm{keV}$ to keep more safely away from the carbon $\mathrm{K}$ edge and from the not so well calibrated effective area region above $2 \mathrm{keV}$. Since the effective area in the now omitted regions is very small, the loss in photon number was unimportant.

The enhanced statistics allowed a further sub-division of the hard band into the $0.5-0.9 \mathrm{keV}(h 1)$ and $0.9-$ $2.0 \mathrm{keV}(h 2)$ bands. Since the diffuse X-ray background is most important below $0.5 \mathrm{keV}$, this enhanced the possibility of finding faint $\mathrm{X}$-ray sources with hard spectra which are invisible in the soft or broad band.

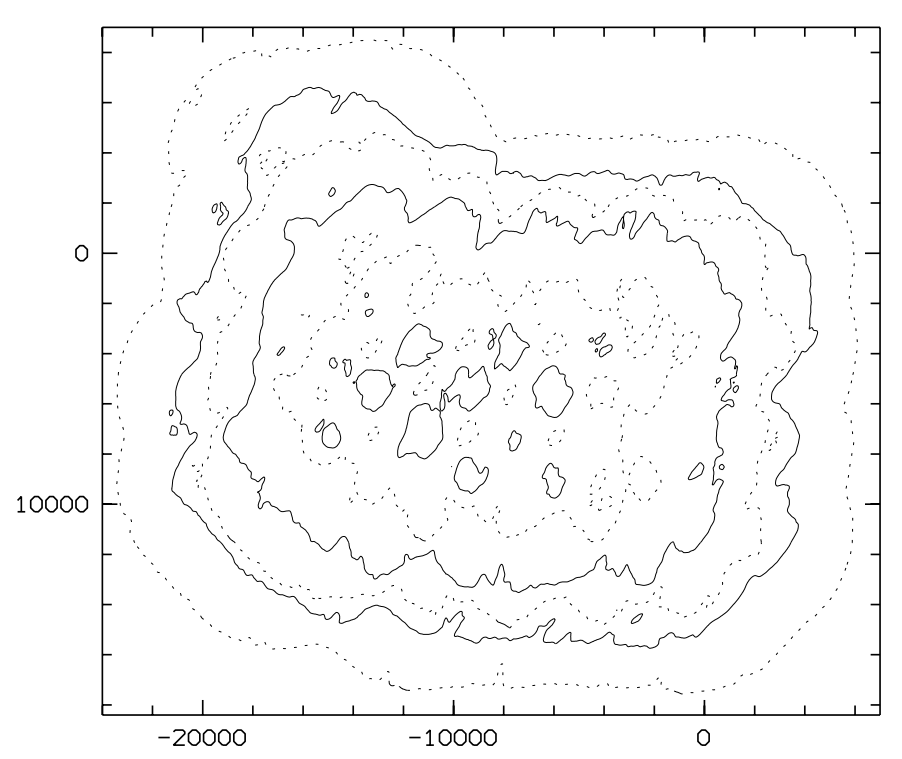

Fig. 3. Merged broad band exposure map of the whole field. The contours mark exposure times of 1 (outermost dotted contour), 5, 8, 13, 20 and 25 (innermost solid contour) ksec, from outside to inside. The axes are in arcseconds, with $9^{\mathrm{h}} 46^{\mathrm{m}} / 48^{\circ} 0^{\prime}(\mathrm{J} 2000.0)$ as zero

The whole image had to be searched now instead of a circular FOV, and the PSF size depended no longer on the distance to the detector centre. Determination of source or background photons had to be weighted by exposure time since strong gradients occured. For that purpose, merged exposure maps were created for the five energy bands. Figure 3 shows the broad band exposure map of the total field. $t_{\exp } \geq 5000 \mathrm{~s}$ was reached for $73 \%$ of the area, and $t_{\text {exp }} \geq 20000 \mathrm{~s}$ for the central $2.3 \mathrm{deg}^{2}$.

Apart from these adjustments, the source detection was performed as described in the previous section. The automatic count rate calculation was unable to handle the complex spatial structure of the merged data. Instead, the exposure time $t_{\exp }$ for each source was read directly from the relevant exposure map. This, together with the number of counts $N_{\text {ct }}$ from the maximum likelihood analysis gave the count rate $c r=N_{\mathrm{ct}} / t_{\text {exp. }}$. Since the exposure maps were already vignetting corrected, no further correction was necessary.

Each source list had to be checked for spurious detections at the pointing borders. When the lists from the five bands were merged, a minimum distance of $3 \sigma$ was required. $410 \mathrm{X}$-ray sources are present in the resulting catalogue, the "merged list". 56 have been detected in the $h 2$ band, and 4, 132, 16, and 202, in the $h 1$, hard, soft, and broad band, respectively. Detection in one band does not necessarily mean that a source is invisible in the others, on the contrary, most sources show $M L_{\text {exi }}>8$ in at least two bands.

\subsection{The $X$-ray catalogue}

The final X-ray catalogue was created by combining the individual catalogue and the merged list. 326 matches between them were found with position differences $\Delta_{\text {im }}$ in the range $0^{\prime \prime} \leq \Delta_{\text {im }} \leq 70^{\prime \prime}$. Due to the complicated PSF structure in the merged data, a minimum distance of $40^{\prime \prime}$ was required for two sources, regardless of $\phi_{\mathrm{oa}}$ of the single pointing detection. 320 pairs had $\Delta_{\text {im }}$ below that limit. The remaining six had $\Delta_{\text {im }}>40^{\prime \prime}$; they were inspected individually to determine whether they are double detections or indeed different sources.

One pair turned out to consist of two real sources. The merged data entry is a $h 2$ band detection, in the single pointing visible only as a faint extension of the other. Four pairs were multiple detections of the same sources. The large $\Delta_{\text {im }}$ were caused by shadowing by the window support structure.

In the last case, the merged list detection is located between two sources found in the same individual pointing, separated by 3.5 . Both sources were of similar magnitude in the detection pointing. In the neighbouring ones, however, the northern source had disappeared, and its average count rate in the merged data was low enough to allow a displacement of nearly $2^{\prime}$ by the influence of the southern source. Therefore, the merged data detection was rejected. Figure 4 shows the distribution of $\Delta_{\text {im }}$ for the accepted double detections.

After the deletion of all double entries, the catalogue contains $574 \mathrm{X}$-ray sources with broad band count rates between $\sim 310^{-3} \mathrm{cts} \mathrm{s}^{-1}$ and $\sim 0.2 \mathrm{cts} \mathrm{s}^{-1}$. Some of the hard band detections were not detected in the broad band. For these, the hard count rate is kept. 324 sources are 


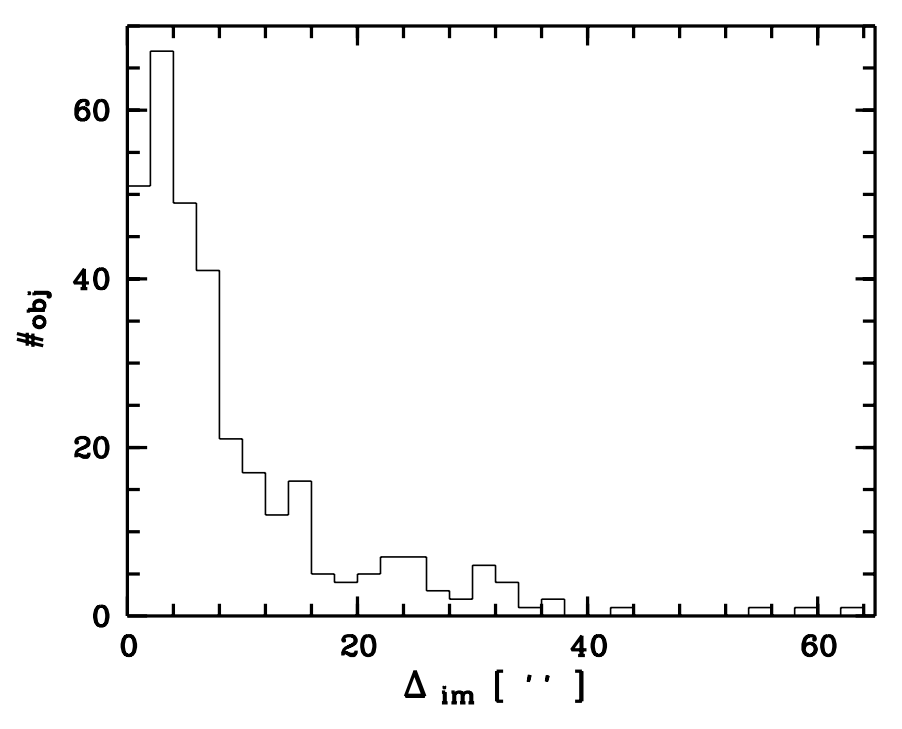

Fig. 4. Distribution of position differences $\Delta_{\text {im }}$ of $\mathrm{X}$-ray sources detected both in individual pointings and the merged data

found in both detection modes, 165 in individual pointings only and 85 in the merged data only. 63 of the latter are found in either of the hard bands with count rates too low to be detected in single pointings.

Of the 165 sources which are detected only in individual pointings, 133 are faint $\left(c r<0.01 \mathrm{cts} \mathrm{s}^{-1}\right)$ objects detected in the central parts of the pointings (i.e. $\phi_{\text {oa }}<20^{\prime}$ ). These are lost when the less sensitive outer parts of the neighbouring pointings are added. The others are located near the borders between pointings or at the edge of the entire field. Variability increases the chance of a source being missed. As was shown by the above example, the average count rate of even rather bright sources might be too low for detection. These results show the necessity of searching both the individual pointings and the merged data. Each mode finds a noticeable amount of sources not detectable in the other.

The final $\mathrm{X}$-ray source catalogue is available as an ascii-file via anonymous ftp at ftp.hs.uni-hamburg.de in the subdirectory pub/outgoing/kmout/data. It is also available at the CDS via anonymous ftp to cdsarc.u-strasbg.fr (130.79.128.5) or via http:// cdsweb.u-strasbg.fr/Abstract.html. The structure of the table is explained in Appendix.

\subsection{Limiting count rates}

A single limiting count rate can be given neither for the individual catalogue nor the merged list. For individual pointings, the differences in exposure time are too large. Even a single limit per pointing is insufficient because of the decreasing sensitivity at larger off-axis angles. Limiting count rates at $\phi_{\mathrm{oa}}=0^{\prime}$ and $\phi_{\mathrm{oa}}=40^{\prime}$, derived with the formulae in Sect. 2.2.1 are included in Table 4.
In the merged data, the net exposure time varies between $\sim 1300 \mathrm{~s}$ at the edges and $>25 \mathrm{ksec}$ in the central parts. The limit in the outermost region is determined by the shortest individual pointing which does not entirely overlap with others (WG 700149 in Table 4). Successive subfields with increasing minimal net exposure time are then selected, the average number of background counts $N_{\mathrm{bg}}$ inside the PSF is measured, and the number of source counts $N_{\mathrm{s}}$ necessary to give $M L_{\text {exi }}=8$ is computed. With an average column density $N_{\mathrm{H}}=10^{20} \mathrm{~cm}^{-2}$, and the assumption of a power law spectrum with energy index $\alpha_{\mathrm{E}}=1$, the limiting count rates correspond to fluxes between $3.410^{-14} \mathrm{ergs} \mathrm{cm}^{-2} \mathrm{~s}^{-1}$ and $2.210^{-13} \mathrm{ergs} \mathrm{cm}^{-2} \mathrm{~s}^{-1}$ in the total ROSAT band. The limits become noticeably fainter for the hard band because the background is much lower above $0.4 \mathrm{keV}$. The hard count rate and flux limits are included in Table 1. It has to be kept in mind, however, that the flux limits in the table are approximations obtained with the assumption of one average spectrum.

Table 1. Limiting count rates for the merged list. $f_{\mathrm{b} / \mathrm{h}, \mathrm{lim}}$ mean limiting fluxes in the broad and hard band, respectively, in units of $10^{-14} \mathrm{ergs} \mathrm{cm}^{-2} \mathrm{~s}^{-1}$. Count rates are given in $10^{-3} \mathrm{cts} \mathrm{s}^{-1}$

\begin{tabular}{|c|c|c|c|c|c|}
\hline $\begin{array}{c}t_{\lim } \\
{[\mathrm{sec}]}\end{array}$ & $\begin{array}{c}\text { area } \\
{\left[\mathrm{deg}^{2}\right]}\end{array}$ & $c r_{\mathrm{b}, \lim }$ & $f_{\mathrm{b}, \lim }$ & $c r_{\mathrm{h}, \lim }$ & $f_{\mathrm{h}, \lim }$ \\
\hline$\simeq 1300^{a}$ & 11.5 & 21.0 & 22.3 & 13.0 & 8.0 \\
\hline 2000 & 10.8 & 16.4 & 17.4 & 10.6 & 6.5 \\
\hline 5000 & 8.3 & 8.5 & 9.0 & 4.2 & 1.9 \\
\hline 10000 & 6.0 & 5.4 & 5.7 & 2.6 & 1.2 \\
\hline 15000 & 4.1 & 4.2 & 4.5 & 2.0 & 0.9 \\
\hline 20000 & 2.4 & 3.5 & 3.7 & 1.7 & 0.8 \\
\hline 25000 & 0.3 & 3.2 & 3.4 & 1.5 & 0.7 \\
\hline
\end{tabular}

${ }^{a}$ : values derived from pointing \#1 in Table 4 .

\section{Additional data}

\subsection{Optical observations in $H S 47.5 / 22$}

The main source of optical information in HS 47.5/22 are the HQS plates. They were taken with the Calar Alto Schmidt telescope on Kodak IIIaJ plates covering the spectral range $3400 \AA-5400 \AA$. Both the direct and spectral plate were scanned with a PDS (Hagen et al. 1995), and the scanned data were used in this work. During the HQS work, 27 optically selected quasars were found in the $11.5 \mathrm{deg}^{2}$ surveyed with ROSAT (filled circles in Fig. 1), 3 of which were previously known.

The faintest sources visible on the objective prism plate have $m_{\mathrm{b}} \lesssim 20^{m}$, while the depth of the direct plate is comparable with the blue plate of the POSS I. X-ray sources with no optical counterpart on the HQS direct 
plate were searched on the red POSS plate, and most of them were found.

\subsection{Radio observations}

\subsubsection{Galactic hydrogen}

As has been noted before, low resolution $\mathrm{H}$ I surveys gave an average value of $\left\langle N_{\mathrm{H}}\right\rangle=10^{20} \mathrm{~cm}^{-2}$ for galactic hydrogen in HS 47.5/22. A new and better resolved map was obtained with the $100 \mathrm{~m}$ RT at Effelsberg ( $9^{\prime}$ beam) to allow individual corrections. The observations were performed during two observing runs in 1989, resulting in a grid with separations $\Delta \alpha=30^{\mathrm{s}}$ and $\Delta \delta=5^{\prime}$, slightly incomplete at the edges.

The measurements are contaminated by stray radiation arising from the sidelobes of the telescope, which can be removed through an all sky deconvolution provided the complete antenna pattern is known. Kalberla et al. (1980) use this method to correct their measurements with the $100 \mathrm{~m}$ RT. Lockman et al. (1986) take a different approach, they combine their spectra to synthetize the beam of the Stark Survey, which is practically uncontaminated (Stark et al. 1992). Any differences between the combined data and the corresponding Stark Survey spectra are attributed to stray radiation present in the former and are therefore subtracted.

Both approaches give a good accuracy but need large amounts of computer time and storage capacity. For the present work, the much easier though less accurate $(20 \%$ in $N_{\mathrm{H}}$ ) time independent correction presented in Kalberla et al. (1980, their Sect. 4.2) is used. They found with several assumptions that the corrected main beam brightness temperature $T_{\mathrm{mb}}$ is

$$
T_{\mathrm{mb}}=T_{\mathrm{A}} \cdot\left(1-\frac{43}{F_{\mathrm{A}}}\right),
$$

where $F_{\mathrm{A}}$ is the observed profile area expressed in $\mathrm{K} \mathrm{km} \mathrm{s}^{-1}$, and $T_{\mathrm{A}}$ is the measured antenna temperature. The small size of HS 47.5/22 means that the distance to the Milky Way does not vary greatly so that it is possible to use one common correction factor. This factor is obtained by dividing $N_{\mathrm{H}}$ values from the Stark Survey by the uncorrected $100 \mathrm{~m}$ RT values at the same positions, and averaging the results:

$$
\left\langle f_{\text {corr }}\right\rangle=0.68 \pm 0.10
$$

Figure 5 shows a contour plot of the corrected column density in HS 47.5/22. Empty grid positions have been filled with the weighted means of the neighbouring positions for the plot.

Variations of a factor $\lesssim 2$, already indicated in the low resolution surveys, could be confirmed on scales of less than $30^{\prime}$. Regions with high $N_{\mathrm{H}}$ can be found near the eastern and along the southern borders of HS 47.5/22 and in the north western edge. The lowest value in the area

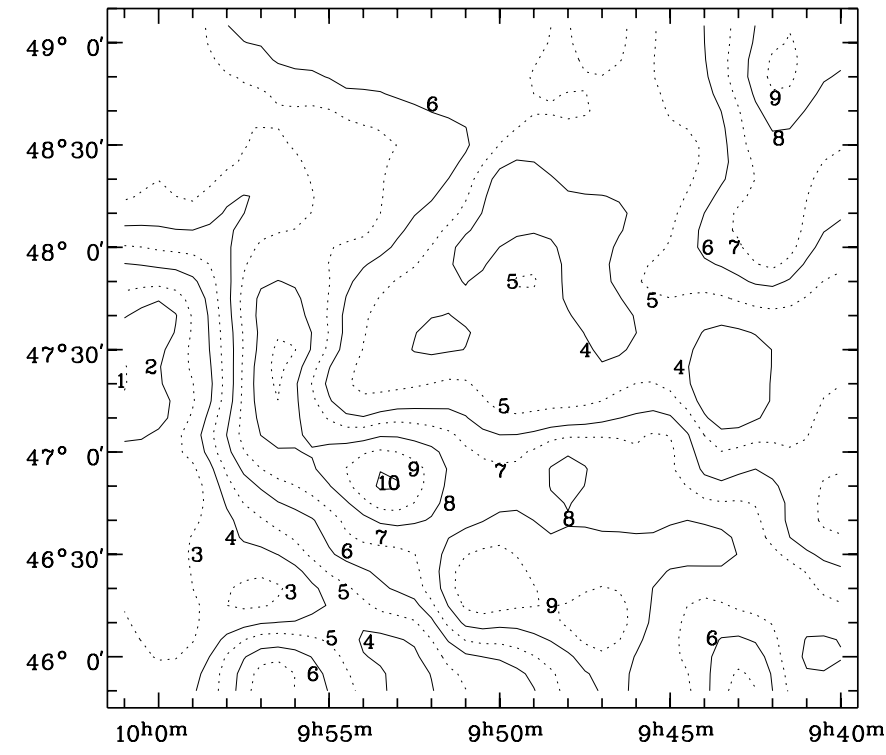

Fig. 5. Contour plot of $N_{\mathrm{H}}$ in HS 47.5/22. Contours are plotted in steps of $5.510^{18} \mathrm{~cm}^{-2}$, starting at $7.910^{19} \mathrm{~cm}^{-2}$ (contour 1). Coordinates are B1950.0

between is less than $910^{19} \mathrm{~cm}^{-2}$. A high velocity cloud found by Hulsbosch \& Wakker (1988) is present in our data as well. It is, however, not the reason for the variations in $N_{\mathrm{H}}$ since the column density peaks of the cloud do not coincide with the overall maxima.

\subsubsection{Radio continuum measurements}

HS 47.5/22 was surveyed with the DRAO interferometer (Penticton/Canada) at $21 \mathrm{~cm}$ and $74 \mathrm{~cm}$ in March 1993. A full analysis of the DRAO observations is under way and will be published elsewhere.

In addition, HS 47.5/22 is located inside the area of several radio continuum surveys which became available during the project. Table 2 gives an overview of position accuracies and flux limits of these surveys. These

Table 2. Overview of Radio surveys covering the field

\begin{tabular}{c|c|cc}
\hline & References & flux limit & pos.err. \\
\hline $87 \mathrm{~GB}(6 \mathrm{~cm})$ & $(1)$ & $18 \mathrm{mJy}$ & $\leq 30^{\prime \prime}$ \\
GB $1.4 \mathrm{GHz}$ & $(2)$ & $100 \mathrm{mJy}$ & $\leq 160^{\prime \prime}$ \\
B3 $408 \mathrm{MHz}^{a}$ & $(3)$ & $100 \mathrm{mJy}$ & $15^{\prime \prime} / 35^{\prime \prime}$ \\
Texas $365 \mathrm{MHz}$ & $(4)$ & $250 \mathrm{mJy}$ & $\approx 5^{\prime \prime}$ \\
DRAO $21 \mathrm{~cm}$ & $(5)$ & $\approx 2 \mathrm{mJy}$ & $\approx 25^{\prime \prime}$ \\
DRAO $74 \mathrm{~cm}$ & $(5)$ & $\approx 30 \mathrm{mJy}$ & $\approx 45^{\prime \prime}$ \\
\hline
\end{tabular}

${ }^{a}$ : covers $\sim 60 \%$ of the field ${ }^{b}$ : errors in $\alpha / \delta$.

${ }^{c}$ : $80 \%$ completeness.

References: (1) Gregory et al. 1996; (2) White \& Becker 1992; (3) Ficarra et al. 1985; (4) Douglas et al. 1996; (5) Wendker et al., in preparation. 
catalogues are crosscorrelated with the $\mathrm{X}$-ray source list. A source is considered identified if the $\mathrm{X}$-ray and radio positions agree within the errors. Only a few $\mathrm{X}$ ray sources match with objets from either catalogue; they are listed in Table 3. Two sources match with $8.4 \mathrm{GHz}$ phase calibration sources in Patnaik et al. (1992); they are included in the table.

Table 3. This table contains the distances between $\mathrm{X}$-ray sources and possible radio counterparts. The first column gives the $\mathrm{X}$-ray sequence number (Table 5 ), the following ones contain the distances $\Delta_{\mathrm{xr}}$ for the surveys (1) - (4) from Table 2 and (5) for Patnaik et al. (1992), and the last column gives the object type

\begin{tabular}{|c|c|c|c|c|c|c|c|}
\hline$\#$ & RX J & $(1)$ & $(2)$ & $(3)$ & $(4)$ & $(5)$ & \\
\hline & $0943.5+4633$ & $3^{\prime \prime}$ & & & & & $\mathrm{D}$ \\
\hline 27 & $0944.6+4638$ & $19^{\prime \prime}$ & & & & & $\mathrm{D}$ \\
\hline 53 & $0945.6+4636$ & $3^{\prime \prime}$ & $8^{\prime \prime}$ & $9^{\prime \prime}$ & $4^{\prime \prime}$ & $5^{\prime \prime}$ & $\mathrm{D}$ \\
\hline 64 & $0945.9+4619$ & $12^{\prime \prime}$ & & & & & gal. cand. \\
\hline 180 & $0950.2+4553$ & $28^{\prime \prime}$ & & & & & BL Lac cand. ${ }^{a}$ \\
\hline 284 & $0953.6+4625$ & $26^{\prime \prime}$ & $40^{\prime \prime}$ & $25^{\prime \prime}$ & & & $\mathrm{D}$ \\
\hline 317 & $0954.7+4608$ & $43^{\prime \prime}$ & & $37^{\prime \prime}$ & & & $\mathrm{D}$ \\
\hline 321 & $0954.8+4715$ & $17^{\prime \prime}$ & & $10^{\prime \prime}$ & & & $\mathrm{D}$ \\
\hline 347 & $0955.6+4721$ & $22^{\prime \prime}$ & & $19^{\prime \prime}$ & & & $\mathrm{D}$ \\
\hline 424 & $0957.7+4848$ & & $60^{\prime \prime}$ & & $23^{\prime \prime}$ & & $\mathrm{D}$ \\
\hline 425 & $0957.7+4745$ & $21^{\prime \prime}$ & & & & & $\mathrm{AGN}^{a}$ \\
\hline 443 & $0958.3+4608$ & $36^{\prime \prime}$ & & & & & $\mathrm{AGN}^{a}$ \\
\hline 446 & $0958.3+4725$ & $4^{\prime \prime}$ & $9^{\prime \prime}$ & $18^{\prime \prime}$ & & $5^{\prime \prime}$ & OK $492^{b}$ \\
\hline 534 & $1000.9+4640$ & $13^{\prime \prime}$ & $29^{\prime \prime}$ & & & & AGN cand. \\
\hline 555 & $1001.5+4659$ & $23^{\prime \prime}$ & & & & & $\mathrm{AGN}_{\text {group }}{ }^{a}$ \\
\hline 571 & $1003.1+4757$ & $28^{\prime \prime}$ & & & & & unident. \\
\hline
\end{tabular}

${ }^{a}$ : identified in this work.

${ }^{b}$ : Peterson et al. (1978).

\section{Optical identification and follow-up observations}

\subsection{Identification with the HQS plates}

Once the catalogue is created, the sources have to be identified. First, the $\mathrm{X}$-ray positions are cross-correlated with the digitised objective prism plate, and all spectra inside the error circles are evaluated. An error radius $R=2 \sigma+10^{\prime \prime}$ is used with the additive term included to allow for systematic errors. $\sigma$ is the standard deviation calculated by the source detection algorithm.

Of all candidates thus selected, $B$-magnitudes are computed from their objective prism spectra which cover the entire Johnson $B$ band. A method was developed (Köhler 1991) which uses an internally determined characteristic curve, calibrated externally with photometric sequences. The spectral information is used to synthesize the
$B$ band. More details are given in Engels et al. (1994). Well above the plate limit, these are accurate to a few tenths of magnitude, with the error rapidly increasing towards fainter objects. Exact $\left(\leq 2^{\prime \prime}\right)$ positions are obtained by identification of the candidates on the direct plate. During this step, obvious artifacts among the spectra are removed, and further optical counterparts below the objective prism plate limit can be found.

Provided the spectra are brighter than $m_{\mathrm{b}} \simeq 18^{\mathrm{m}} 5$, a classification is possible: early stars can be distinguished from late or very late ones, galaxies and AGN are usually recognizable. At $m_{\mathrm{b}} \geq 18.5$, only the raw forms of the spectra can be seen; they are classified as weak and extremely blue (these are usually faint AGN), blue (again, many of these are AGN, although the fraction of nonAGN grows) or red. For a certain amount of sources, no classification is possible, e.g. saturated spectra or overlaps. Examples of the respective objective prism spectra and a detailed description of this identification step are given in Bade et al. (1997).

All optical counterparts thus found are cross-checked with published catalogues (Véron-Cetty \& Véron 1996; SIMBAD $^{1}, \mathrm{NED}^{2}$ ) and the HQS archive. 22 objects from the original optical quasar sample could be identified as $\mathrm{X}$-ray sources. In total, the following results were obtained:

24 known AGN,

14 known stars,

2 clusters of galaxies (one candidate),

51 AGN candidates (classified as AGN or extremely blue),

21 galaxy candidates,

50 stellar candidates (including saturated spectra),

23 faint blue objects,

17 faint red objects,

19 unidentified spectra (including overlaps),

270 visible on the HQS direct plate only,

83 empty fields (i.e. no counterpart on the HQS plate).

Several error circles contain more than one optical counterpart. For the determination of the most plausible one, all information is put together. The ratio of $\mathrm{X}$-ray to optical flux $\log \left(f_{\mathrm{x}} / f_{\mathrm{b}}\right)$ is crucial in cases where both stellar and non-stellar objects are found; the former have mostly $\log \left(f_{\mathrm{x}} / f_{\mathrm{b}}\right)<-1$ while the latter have $\log \left(f_{\mathrm{x}} / f_{\mathrm{b}}\right)>-1$. Raw spectral information could be helpful in classifying white dwarfs (very soft X-ray spectra) or galaxy clusters (hard X-ray spectra). In those cases where a known AGN

1 Set of Identifications, Measurements and Bibliography for Astronomical Data; operated by the Centre de Données astronomiques de Strasbourg.

2 NASA/IPAC Extragalactic Database, operated by the Jet Propulsion Laboratory, California Institute of Technology under contract with the National Aeronautics and Space Administration. 
falls inside the error circle, it has been automatically taken as the proper identification. Some X-ray sources in the field HS 47.5/22 have been previously detected, either in the RASS (Bade et al. 1995; Voges, private communication) or with Einstein. For these, the published identification is used.

\subsection{Follow-up spectroscopy}

Follow-up observations were performed during a 5 nights period in March 1994 with the $3.5 \mathrm{~m}$ telescope at Calar Alto/Spain. Candidates were selected by decreasing X-ray count rate in the attempt to obtain a flux limited quasar sample. X-ray sources with obvious stellar identifications were ignored, and some special sources with lower count rates were included .

The $3.5 \mathrm{~m}$ telescope was equipped with the focal reducer (f/2.7) and a TEK CCD $(1024 \times 1024$ pix, $24 \mu /$ pix $)$. With this instrumentation it is possible to switch between direct imaging and spectral mode. Due to nonphotometric weather and moderate seeing $\left(\sim 2^{\prime \prime}\right)$, a rather wide slit ( $\left.3^{\prime \prime} 3\right)$ had to be used. For direct imaging, Johnson $B V R$ filters were mounted.

Since many candidates were faint $\left(m_{\mathrm{b}} \gtrsim 19.5\right)$, and since our foremost interest was redshift determination, the low-dispersion grism ( $905 \AA / \mathrm{mm})$ was used to obtain a sufficient signal to noise in the shortest possible time. The large wavelength range thus covered $(3600 \AA-9000 \AA)$ includes normally at least two emission lines, so that the resulting redshifts are accurate to better than 0.01. Line shape analysis, on the other hand, is practically impossible with this low resolution (FWHM $50 \AA$ ). Acquisition exposures were made with the $B$-filter to obtain better magnitudes than those derived from the objective prism plates.

Spectra of a He lamp have been taken to get an absolute wavelength scale. In a few cases, the wavelength calibration was corrected with the sky absorption lines at $6888 \AA$ and $7594 \AA$. Spectrophotometric and photometric standards were observed two or three times per night for flux and magnitude calibration. However, due to nonphotometric weather condition, no absolute flux calibration could be achieved.

54 X-ray sources could be observed. Five of them had no optical counterparts on the HQS plates, all of them hard band detections. They might therefore be distant clusters of galaxies or high redshift quasars, both unlikely to appear on blue plates. One minute red exposures were taken in search of counterparts, with a limit of $m_{\mathrm{R}} \simeq 22 .{ }^{\mathrm{m}} 5$. Two exposures showed an object inside the respective $\mathrm{X}$-ray error circles, of which spectra were obtained. One could be identified with a $z=1.240$ quasar (RX J 0948.5+4751), the other (RX J 0958.3+4702) with a galaxy at $z=0.39$. Although the missing counterparts and thus extreme $\log \left(f_{\mathrm{x}} / f_{\mathrm{b}}\right)$ made the remaining three

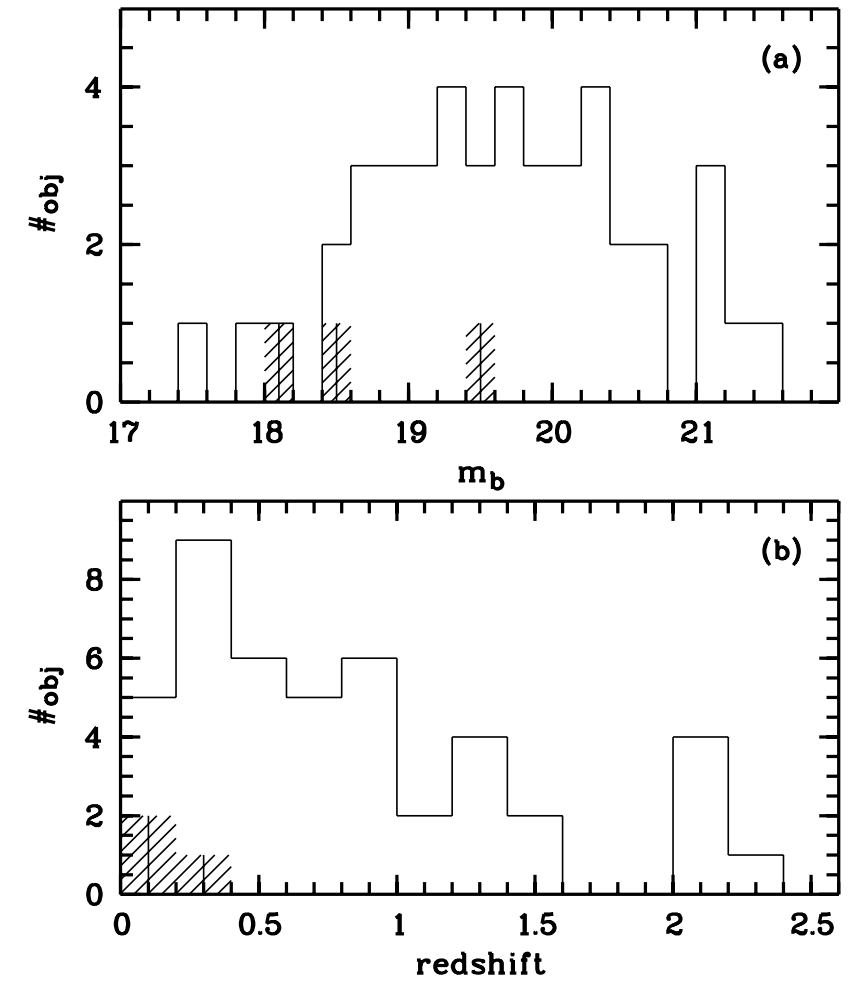

Fig. 6. $B$ magnitude a) and redshift b) distribution of the newly identified AGN. The emission line galaxies and the group (counted as one) are shaded

sources even more interesting, deeper searches had to be omitted in favour of identifying other objects.

$45 \mathrm{X}$-ray sources are clearly extragalactic. One is the galaxy mentioned above, another is a group of three more or less active galaxies, 41 are AGN. For the remaining two cases, the identification is ambiguous. Both spectra show emission lines on top of a galaxy continuum, but neither resolution nor signal to noise is sufficient to reveal any further details on the object types. These two sources are marked as "ELG" in Table 6.

Three objects have featureless slit spectra. Their objective prism spectra resemble those of emission line AGN at similar apparent magnitudes, so a classification as "BL Lac candidate" seems plausible. For one object, this possibility is supported by radio data: RX J $0950.2+4553$ has been detected at $6 \mathrm{~cm}$ (Gregory et al. 1996), $21 \mathrm{~cm}$ and $74 \mathrm{~cm}$. The other two, however, appear to be radio quiet. Their ratio of $\mathrm{X}$-ray to optical flux is moderate compared with Nass et al. (1996), who found that many X-ray selected BL Lac objects have $\log \left(f_{\mathrm{x}} / f_{\mathrm{b}}\right) \geq 1.8$. Although neither missing radio detections nor moderate $\log \left(f_{\mathrm{x}} / f_{\mathrm{b}}\right)$ $(<0.6$ for both $)$ rules out the BL Lac hypothesis entirely, these facts argue against it. Therefore both objects are denoted "unidentified", although it can be safely claimed by the featureless spectra that they are no ordinary AGN.

The last three objects are stars. The low resolution of the spectra allows no classification beyond the 
recognition of two $\mathrm{M}$-stars which are easily recognizable by their broad absorption bands.

Table 6 lists the observed objects, their final identification, $B$-magnitude and redshift (where available). The AGN spectra are presented in Fig. 7, and redshift and magnitude distribution of the AGN are shown in Figs. 6a and b.

One additional object was observed by N. Bade with the Russian 6m telescope near Selenchukskaya, covering the wavelength range $3600 \AA-7200 \AA$. This object had originally been selected as AGN candidate in the HQS but was misidentified during their follow-up observations. The new spectrum is shown at the end of Fig. 7. One emission line is clearly visible above the noise. Associating it with MgII 2798 gives a redshift $z=0.77$. The absence of other lines supports this since at that redshift, strong emission lines falls into the observed spectral range.

\section{Concluding remarks}

The X-ray catalogue derived from the medium deep ROSAT Survey in HS 47.5/22 contains in total 574 X-ray sources with likelihoods $M L_{\text {exi }}>8$ and broad band fluxes $f_{\mathrm{x}}>710^{-15} \mathrm{ergs} \mathrm{cm}^{-2} \mathrm{~s}^{-1}$.

The analyses of both individual pointings and merged data were proven to be necessary to find all the $\mathrm{X}$-ray sources in the field. As a completeness test for the cata$\operatorname{logue}$, the $\log N / \log S$-curve was created and compared with the literature (Molthagen 1996). We found that for sources with $M L>10$, our catalogue is of comparable completeness with previous works down to a broad band flux $f_{\mathrm{b}} \approx 3.410^{-14} \mathrm{ergs} \mathrm{cm}^{-2} \mathrm{~s}^{-1}$; details of this and other results (e.g. variability) will be given elsewhere (Molthagen, in preparation).

Acknowledgements. We wish to thank the HQS group for their readiness to share experience and software, and especially for giving access to their data prior to publication. KM wishes to thank N. Bade for his help with the follow-up observations, and the MPE Garching for the possibility to perform the merging of the X-ray pointings, and analysis thereof, in Garching. We acknowledge that $\mathrm{C}$. Feldt performed and reduced the $100 \mathrm{~m}$ RT $21 \mathrm{~cm}$ line observations.

This work has made use of the NASA/IPAC Extragalactic Database (NED), which is operated by the Jet Propulsion Laboratory, California Institute of Technology, under contract with the National Aeronautics and Space Administration.

Part of this work was supported by a grant from the BMFT (DARA FKZ 50 OR 9308).

\section{References}

Bade N., 1993, PhD Thesis, Hamburg University Bade N., Engels D., Voges W., et al., 1997, A\&AS (accepted)
Bade N., Fink H.H., Engels D., et al., 1995, A\&AS 110, 469 Becker R.H., White R.L., Edwards A.L., 1991, ApJS 75, 1 Boyle B.J., Shanks T., Georgantopoulos I., Stewart G.C., Griffiths R.E., 1994, MNRAS 271, 639

Cruddace R.G., Hasinger G.R., Schmitt J.H., 1988, in Murthag F., Heck A. (eds.) ESO Conf. Workshops Proc. 28

Douglas J.N., Bash F.N., Bozyan F.A., Torrence G.W., Wolfe C., 1996, AJ 111, 1945

Engels D., Cordis L., Köhler T., 1994, in MacGillivray H.T., Thomson E.B., Lasker B.M. et al. (eds.) IAU Symp. 161, 317

Engels D., Hagen H.-J., Cordis L., et al., 1997 (in press)

Ficarra A., Grueff G., Tomassetti G., 1985, A\&AS 59, 255

Gioia I.M., Maccacaro T., Schild R., et al., 1990, ApJS 72, 567

Giommi P., Tagliaferri G., Angelini L., 1988, Mem. Soc. Astr. Italiana 59, 33

Gregory P.C., Scott K.W., Douglas K., Condon J.J., 1996, ApJS 103, 427

Hagen H.-J., Groote D., Engels D., Reimers D., 1995, A\&AS 111,195

Hasinger G., Burg R., Giacconi R., et al., 1993, A\&A 275, 1

Hulsbosch A.N.M., Wakker B.P., 1988, A\&AS 75, 191

Kalberla P.M.W., Mebold U., Reich W., 1980, A\&A 82, 275

Klemola A.R., Jones B.F., Hanson R.B., 1987, AJ 94, 501

Köhler T., 1991, Diploma Thesis, Hamburg University

Lockman F.J., Jahoda K., McCammon D., 1986, ApJ 302, 432

Molthagen K., 1996, PhD thesis, Hamburg University

Molthagen K., Wendker H.J., Briel U.G., 1994, IAU symp. 159, 517

Morrison R., McCammon D., 1983, A\&A 270, 119

Nass P., Bade N., Kollgaard R.I., et al., 1996, A\&A 309, 419

Notni P., 1980, Astron. Nachr. 301, 51

Patnaik A.R., Browne I.W.A., Wilkinson P.N., Wrobel J.M., 1992, MNRAS 254, 655

Peterson B.M., Craine E.R., Strittmatter P.A., 1978, PASP 90, 386

Pfeffermann E., Briel U.G., Hippmann H., et al., 1986, Proc. SPIE 733, 519

Puchnarewicz E.M., Mason K.O., Cordova F.A., et al., 1992, MNRAS 256, 589

Schmidt M., Schneider D.P., Gunn J.E., 1986, ApJ 310, 518

Schneider D.P., Schmidt M., Gunn J.E., 1994, AJ 107, 1245

Stark A.A., Gammie C.F., Wilson R.W., et al., 1992, ApJS 79, 77

Stocke J.T., Morris S.L., Gioia I.M., et al., 1991, ApJS 76, 813

Trümper J., 1983, Adv. Space Res. 2, 241

Véron-Cetty M.-P., Véron P., 1996, ESO Sci. Rep. No. 17

Voges W., 1992, in Proc. of the ISY Conference Space Science, ESA ISY-3. ESA Publications

White R.L., Becker R.H., 1992, ApJS 79, 331

Zimmermann H.U., Belloni T., Izzo C., Kahabka P., Schwentker O., 1993, EXAS User's Guide, MPE report 244

Zimmermann H.U., Becker W., Belloni T., et al., 1994, EXAS User's Guide, MPE report 257 